\title{
En relación con el artículo "Dispensación de fentanilo y tapentadol en farmacia comunitaria: adherencia farmacoterapéutica en dolor no oncológico"
}

\author{
José Espejo Guerrero \\ Farmacéutico comunitario en Adra (Almería).
}

\section{Sr. Director:}

Hemos leído con interés el artículo "Dispensación de fentanilo y tapentadol en farmacia comunitaria: adherencia farmacoterapéutica en dolor no oncológico", de Reyes Estévez y colaboradores, publicado en la revista que usted dirige (1). En el mismo se analiza la adherencia terapéutica a estos dos principios activos en dolor crónico no oncológico (DCNO), sus causas y sus consecuencias, aportando interesantes datos al respecto. Observamos que el estudio ha supuesto un enorme trabajo de campo, con la participación de 139 farmacéuticos comunitarios. Queremos por ello enriquecer, si es posible, los resultados encontrados en dicho artículo, a la vez que contrastar ciertos datos.

Respecto a la composición de la muestra del presente el estudio, se trata, como señalan los autores, de una submuestra de la del estudio previo publicado con esta misma base de datos (2). Concretamente, de los 559 del estudio anterior se seleccionan 478 con dolor crónico no oncológico (DCNO) a la que dicen los autores se referirán en el presente estudio. Pero no es así, pues finalmente utilizan 411, ya que los 67 restantes son tratamientos de inicio, en los que, obviamente, no se puede determinar la adherencia. Es por ello que creemos que la tabla 1, que describe edad y sexo, lo debe hacer de los 411 pacientes del presente estudio y no copiar, como han hecho, la tabla 1 del estudio anterior. Esta circunstancia afecta al análisis de los datos y será comentada más adelante.

Se ha observado un grado de adherencia del 87,1\%. Creemos que aquí sí es interesante indicar en el intervalo de confianza del 95\%, pues es la adherencia el parámetro principal que se intenta determinar. Hemos calculado que dicho intervalo está comprendido entre el 83,5\% y el $90,2 \%(3)$.

Se valora la influencia de cuatro variables en el grado de adherencia: el principio activo, la forma farmacéutica, la edad y el sexo (tablas 2,3, 4 y párrafo $6^{\circ}$ del apartado Resultados).
La tabla 2 es confusa. A lo largo del trabajo de Reyes $\mathrm{D}$, se incluye una línea que contiene aquellos pacientes no incluidos en el estudio por no cumplir los criterios de inclusión, que creemos no deberían figurar en ninguna de las tablas del mismo. Dicha tabla quedaría de la siguiente forma:

\begin{tabular}{|l|c|c|}
\hline \multicolumn{1}{|c|}{$\mathbf{n = 4 1 1}$} & $\begin{array}{c}\text { Fentanilo } \\
(\mathrm{n}=\mathbf{2 3 5} ; \mathbf{5 7 , 2} \%)\end{array}$ & $\begin{array}{c}\text { Tapentadol } \\
(\mathrm{n}=\mathbf{1 7 6} ; \mathbf{4 2 , 8} \%)\end{array}$ \\
\hline Si adherentes & $195(83 \%)$ & $163(92,6 \%)$ \\
\hline No adherentes & $40(17 \%)$ & $7,4 \%)$ \\
\hline
\end{tabular}

Siendo la diferencia de un principio activo a otro, en cuanto a grado de no adherencia, estadísticamente significativa: $17,0 \%$ en fentanilo vs 7,4 para el tapentadol $(d=9,6$; IC 95\% entre 3,5 y 15,8\%; p=0,004).

En cuanto a la forma farmacéutica, la no adherencia está clarísimamente relacionado con los parches transdérmicos, responsables del $100 \%$ de la no adherencia del fentanilo.

Respecto a la edad, al no tener una tabla 1 que describa la muestra estudiada, hemos de suponer que sigue la proporción en cuanto a edad y sexo de dicha tabla del estudio anterior, de modo que de los 411, 226 serán mayores de 70 años ((307/559) x 411). Se indica en el párrafo $6^{\circ}$ de Resultados que el 77\% de los incumplidores son mayores de 70 años. Entonces:

El $77 \%$ de los incumplidores (41 de los 53 incumplidores) son mayores de 70 años.

\begin{tabular}{|l|l|c|c|}
\hline Edad (años) & \multicolumn{1}{|c|}{ No adherentes } & Adherentes & Total \\
\hline Mayor de 70 & $\begin{array}{l}41 \text { (el } 18,1 \% \text { de los } \\
\text { mayores) }\end{array}$ & 185 & 226 \\
\hline $\begin{array}{l}\text { Menor o } \\
\text { igual a } 70\end{array}$ & $\begin{array}{l}12 \text { (el 6,5\% de los } \\
\text { menores) }\end{array}$ & 173 & 185 \\
\hline
\end{tabular}

Diferencia $(18,1 \%$ vs $6,5 \%)$, que si es estadisticamente significativa ( $d=11,6 \%$; IC95 \% entre 5,5 y $17,8 \% ; p=0,0005)$. 
En cambio, con la variable sexo, al contrario de lo que parece en el texto, no influye en la adherencia. En efecto, en el mismo párrafo se dice que el $79 \%$ de los incumplidores (es decir 42 de los 53 incumplidores) son mujeres. Haciendo un razonamiento similar al anterior en cuanto a la composición de la muestra, habrá 284 mujeres y 127 hombres.

\begin{tabular}{|c|l|c|c|}
\hline Sexo & \multicolumn{1}{|c|}{ No adherentes } & Adherentes & Total \\
\hline Mujer & $\begin{array}{l}42 \text { (el 14,8\% } \\
\text { de las mujeres) }\end{array}$ & 242 & 284 \\
\hline Hombre & $\begin{array}{l}11 \text { (el 8,7\% } \\
\text { de los hombres) }\end{array}$ & 116 & 127 \\
\hline
\end{tabular}

Diferencia ( $14,8 \%$ vs $8,7 \%)$, que no es estadísticamente significativa $(p=0,09)$.

En cuanto a la valoración de la efectividad, se evalúa la influencia de la adherencia por un lado (tabla 5) y del principio activo por otro (tabla 6), pero con variables resultado diferentes (escala Eva en el primer caso y sensaciones del paciente en el segundo). Aún con la imposibilidad de hacer un análisis estratificado o multivariante, para aislar la influencia de estas dos importantes variables (la adherencia que es el objeto del estudio, y el principio activo, que es muy importante), algo podemos aportar a los resultados descritos.

Respecto a la valoración de la influencia de la adherencia en la efectividad del tratamiento, se indica en la tabla 5 que la adherencia no influye en la percepción de dolor (6,01 en adherentes vs 6,6 en no adherentes; $p=0,097)$. Pero esta no diferencia puede deberse a una falta de sensibilidad de la escala de medida, como sugieren los autores, o a un tamaño de muestra no suficiente. En efecto, con el tamaño de la muestra con la que se ha trabajado, hemos hallado que el estudio tiene una potencia del 39\% para encontrar una diferencia significativa, si es que realmente la hay; muy lejos del 80\% mínimo deseable. Para alcanzar esta potencia, hemos calculado que haría falta un tamaño de muestra de 983 sujetos. Viene ello a poner de manifiesto la necesidad del cálculo previo del tamaño de muestra necesario, cuestión que desconocemos si se ha tenido en cuenta en el protocolo del presente estudio.

Sobre la influencia del principio activo en las mejoras percibidas por el paciente, cuestión crucial de la efectividad percibida, tenemos aquí algunas desavenencias con los resultados expresados por los autores. En la tabla 6 se hace referencia a este apartado, diciéndosenos al respecto en el texto:

"los pacientes con dolor no oncológico consideran sí tener el dolor controlado en un $41 \%$, para un $53 \%$ sí ha mejorado su capacidad funcional y para un 57\% sí se ha mejorado su calidad de vida”.

Lo cual no es cierto según los datos. En la tabla 6 sobran las columnas de "no se les pregunta", pues corresponden a los 67 casos de tratamientos de inicio. Por tanto, la tabla correcta para evaluar la efectividad de los tratamientos es la siguiente:

\begin{tabular}{|l|c|c|c|c|c|c|}
\hline & \multicolumn{2}{|c|}{ Fentanilo } & \multicolumn{2}{c|}{ Tapentadol } & \multicolumn{2}{c|}{ Total } \\
\hline & No & Si & No & Si & No & Si \\
\hline $\begin{array}{l}\text { Controlado el } \\
\text { dolor crónico }\end{array}$ & 109 & 127 & 106 & 71 & 215 & 198 \\
\hline $\begin{array}{l}\text { Mejora } \\
\text { capacidad } \\
\text { funcional }\end{array}$ & 81 & 155 & 77 & 99 & 158 & 254 \\
\hline $\begin{array}{l}\text { Mejora calidad } \\
\text { de vida }\end{array}$ & 69 & 165 & 68 & 107 & 137 & 272 \\
\hline
\end{tabular}

Por tanto, el 47,9\% ((198/(198+215)) de los pacientes sí controlan el dolor crónico, el 61,7\% sí mejora su capacidad funcional y el 66,5\% sí mejora su calidad de vida. Pero si profundizamos en el análisis, como se propone al distribuir los resultados por principio activo, observamos que más de la mitad de los pacientes que usan fentanilo controlan su dolor crónico (el 53,8\% (=127/(127+109)), control que ocurre con menos de la mitad de los que usan tapentadol (el 40,1\%(=71/ $(71+106))$. Y esta diferencia de efectividad sentida sí es estadísticamente significativa a favor del fentanilo $(\mathrm{d}=13,7 \%$; IC95\% entre 4,1 y 23,3\%; p=0,006). Y ello sin tener en cuenta la menor adherencia con el fentanilo, por lo que creemos que si pudiésemos aislar los efectos de las variables principio activo y adherencia, por ejemplo con una regresión logística, la diferencia de efectividad sería aún mayor a favor del fentanilo. También lo es para la mejora de la calidad de vida $(d=9,4 \%$; IC95\% entre 0,08 y $18,7 \% ; p=0,047)$ y casi para la mejora de la capacidad funcional $(p=0,052)$. Y estos datos sí nos parecen relevantes e importantes.

Nada podemos decir sobre la influencia de las diferentes variables recogidas sobre la seguridad, pues nada se dice al respecto.

Viene todo ello a colación de la pregunta que hace unos días me hizo una paciente mayor de 70 años usuaria de tapentadol en comprimidos, cuestionándome sobre "si los parches para el dolor serían mejores que las pastillas que tomaba” y en seguida me vino a la cabeza el artículo que estamos comentando.

\section{Referencias bibliográficas}

1. Reyes D, González A, Merencio E, Córcoles ME. Dispensación de fentanilo y tapentadol en farmacia comunitaria: adherencia farmacoterapéutica en dolor no oncológico. Farmacéuticos Comunitarios. 2021 Jan 20; 13 (1): 32-59. doi:10.33620/ FC.2173-9218.(2021/Vol13).001.06

2. González A, Reyes D, Merencio E, Córcoles M.E. Dispensación de fentanilo y tapentadol en farmacia comunitaria: perfil del paciente e indicación terapéutica en dolor crónico no oncológico. Farmacéuticos Comunitarios. 2018 Sep 28; 10(3):25-31. doi:10.5672/FC.2173-9218.(2018/Vol10).003.04

3. Cálculos realizados con la ayuda del paquete estadístico Stata 15.1. StataCorp LLC. 


\section{Respuesta a José Espejo Guerrero}

Querido José Espejo:

En primer lugar, muchas gracias por haber leído nuestros artículos sobre opioides. Es un tema muy controvertido y que nos genera gran preocupación en la farmacia comunitaria.

En segundo lugar, gracias por hacer una revisión tan exhaustiva. Hablo en nombre de mis compañeros coautores cuando te digo que nos encanta la crítica constructiva y más si es con ánimo de que todos mejoremos cada día.

Con respecto a la cuestión que nos atañe, una vez leída y reflexionada, queremos hacer varias puntualizaciones:

- Por lo que veo tienes una propuesta diferente a la nuestra. Seguramente puede que sea hasta incluso más acertada.

- Respecto a la línea que comentas sobre las tablas, podríamos haberlas quitado, pero considerábamos que se entendía que eran pacientes de inicio. Por otro lado, queríamos plasmar una descripción de la muestra completa por darle más relevancia y recordar al lector de dónde parte el estudio.
- En lo que no estamos de acuerdo es que no cumplan los criterios de inclusión. Los cumplen, pero como no tienen tratamiento de continuación no evaluamos adherencia. Con esto queríamos mostrar que había pacientes del estudio con tratamiento de inicio.

Por último, como has mostrado gran inquietud con el mundo de los opioides, queremos también invitarte al grupo del Dolor de SEFAC como miembros y coordinadores que somos. Y a que participes en otros proyectos de investigación que tenemos en mente, donde tu experiencia y saber estadístico nos serían de gran ayuda.

Si quieres hacernos llegar alguna opinión más, doy permiso al director de la revista a que te facilite mi correo electrónico y así podamos mantener un diálogo más amplio. De otro modo, también podemos intercambiarnos mensajería instantánea a través del grupo que compartimos todos.

Un abrazo y gracias.

Daniel Reyes Estévez, en nombre de los autores. 